\title{
Period04: A software package to extract multiple frequencies from real data
}

\author{
Patrik Lenz and Michel Breger \\ Institut for Astronomy, Vienna University, Türkenschanzstrasse 17, A-1180 Vienna, Austria \\ e-mail: lenz@astro.univie.ac.at
}

\begin{abstract}
Period04, a reworked and extended version of Period98 (Sperl 1998) and PERIOD/ PERDET (Breger 1990), is a new software package especially dedicated to the statistical analysis of large astronomical data sets containing gaps. It offers tools to extract the individual frequencies from the multiperiodic content of time series and provides a flexible interface to perform multiple-frequency fits. A review of the functions of Period04 is given.
\end{abstract}

Keywords. Methods: data analysis, methods: numerical

\section{Introduction}

The Vienna Asteroseismology Group continues to develop reduction packages for astronomical time series. PERIOD/PERDET and Period98 have already been made available for public download. Both packages contain computer programs to search for and fit sinusoidal patterns within time series of data in which one suspects periodic behavior. Period98 provides a flexible graphical interface for least-squares calculations, which has proved to be very convenient for the work of our group.

Over the years we noticed that new users are often deterred by the seeming complexity of the Period98 interface. Consequently, a cleaner interface as well as a number of new functions needed to be added.

For this reason Period04, a revised version of Period98, has been developed. The program takes advantage of a combination of scanning single-frequency techniques (Fourier as well as reduction of residuals) and multiple-frequency analyses (least-squares fitting to the data), including the possibility to examine amplitude and phase variations. The minimisation of the residuals of sinusoidal fits to the data is based upon the CURFIT routine from Bevington (1969).

\section{Rework of the graphical user interface}

The graphical user interface of the program has been rewritten in Java, a programming language that provides very powerful tools to create platform independent user interfaces.

Due to performance reasons the management of the data and the calculations are implemented in $\mathrm{C}++$. Thus, Period04 is a Java/C++ hybrid program.

To provide more user convenient features such as shortcuts for frequently used commands, tool-tips and pop-up menus to edit list items have been introduced. Furthermore, plots can be exported in eps and jpg formats. Also, the labels of the axes can be edited.

Moreover, the help system has been reworked and is now more problem-oriented. Specific tutorials introduce the user to the package by means of several example time strings. 


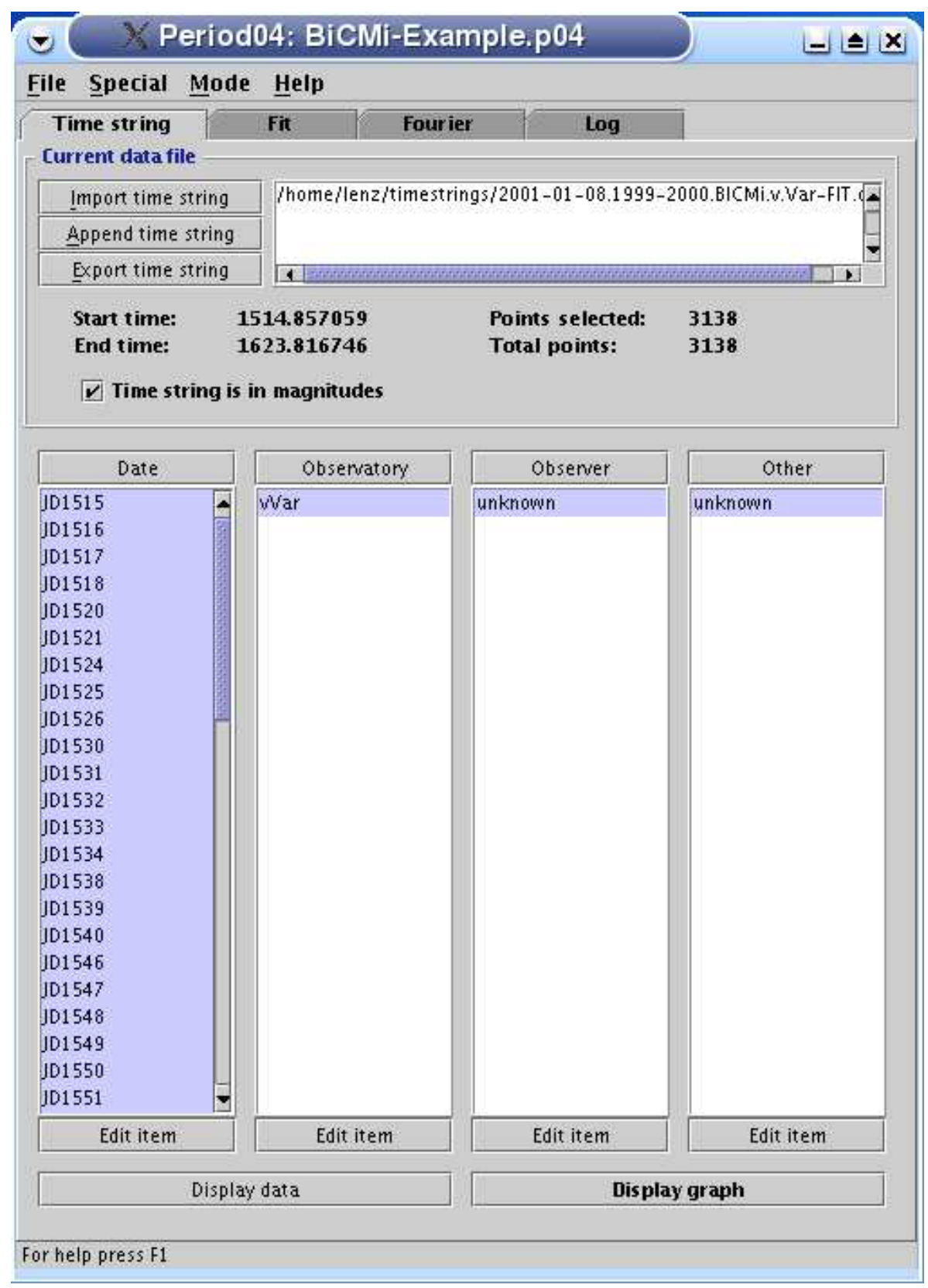

Figure 1.

\section{New features}

Apart from the improvements of the graphical interface the program has been extended to offer a special calculation mode to fit data containing a periodic time shift, e.g., to search for light-time effects in binary systems.

In addition, the program now displays the formal uncertainties for the calculated leastsquares fit for both calculation modes. 


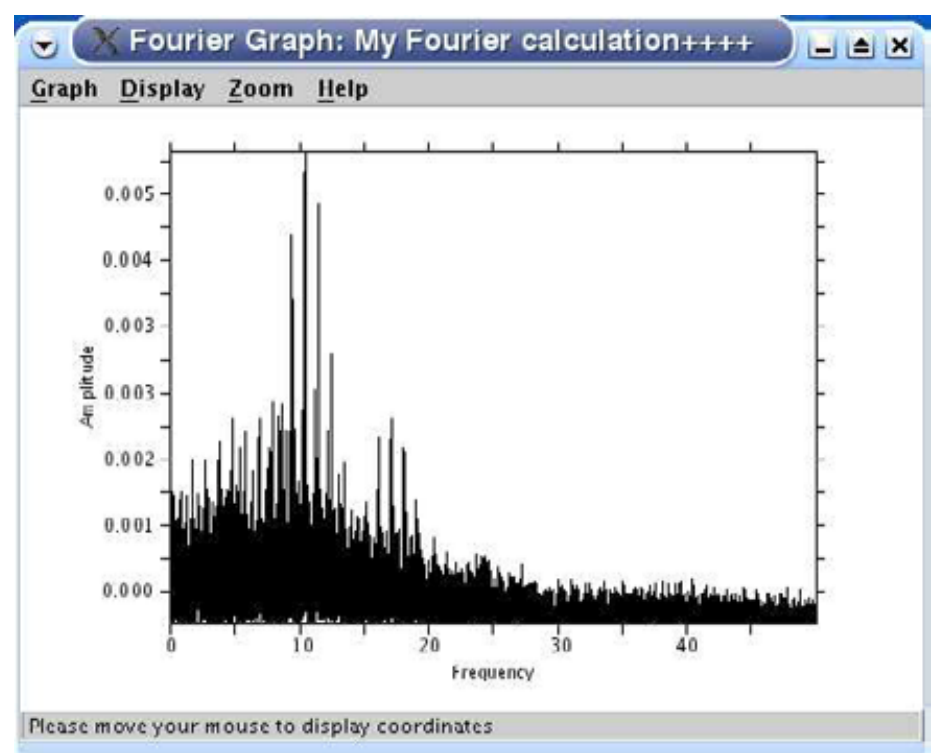

Figure 2.

To meet the demands of different fields of research, preferred default values for the Fourier calculation and other settings can now be defined in a preferences file.

\section{Future extensions}

We intend to implement a three-dimensional visualisation tool which will provide some indication of the interdependencies of frequency peaks caused by aliasing. Moreover, we

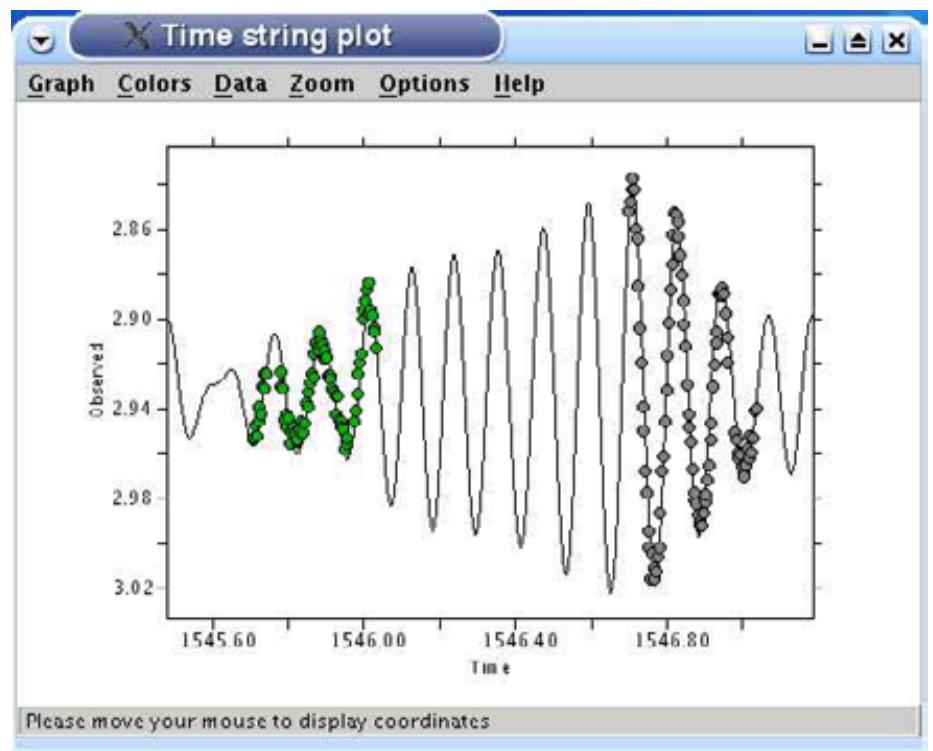

Figure 3. 


\begin{tabular}{|c|c|c|c|c|c|}
\hline \multicolumn{4}{|c|}{ - 3 Period04: test.p04 } & \multirow{2}{*}{\multicolumn{2}{|c|}{ 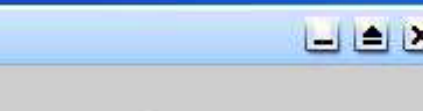 }} \\
\hline File Special & Mode & Hel & & & \\
\hline Time string & & Fit & Fourier & \multicolumn{2}{|c|}{$\log$} \\
\hline \multicolumn{2}{|c|}{ Periodic time shifts } & \multicolumn{2}{|c|}{ Goodness of Fit } & & \\
\hline \multicolumn{3}{|c|}{ Import frequencies } & \multirow{2}{*}{\multicolumn{2}{|c|}{$\begin{array}{l}\text { Selected Frequencies: } \\
\text { Zero point: } \\
\text { Residuals: }\end{array}$}} & \multirow{2}{*}{$\begin{array}{l}12 \\
-10.3774749 \\
2.74189444\end{array}$} \\
\hline Export freq & quencies & & & & \\
\hline \multicolumn{6}{|c|}{ Settings for Least Square Fit Calculation } \\
\hline \multicolumn{3}{|c|}{ Fitting Formula: } & \multicolumn{3}{|c|}{$Z+\Sigma A_{i} \sin \left(2 \pi\left(\Omega_{i}\left[t+\alpha_{p t s} \sin \left(2 \pi\left(w_{p t s} t+\varphi p t s\right)\right]+\phi_{i}\right)\right)\right.$} \\
\hline \multicolumn{3}{|c|}{ Calculations based on: } & () Original d & \multicolumn{2}{|c|}{ Adjusted data } \\
\hline Use weights: & & none & Edit weigh & & \\
\hline
\end{tabular}

\begin{tabular}{|c|c|c|c|c|}
\hline \multicolumn{4}{|c|}{ Parameters for the periodic time shift: } & 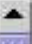 \\
\hline \multirow{3}{*}{ (1) PTSF } & Frequency & Amplitude & Phase & \\
\hline & 0.00676235757 & 0.000776178295 & 0.598334 & \\
\hline & \multicolumn{2}{|c|}{ Search PTS start values } & Improve PTS & \\
\hline Use Freq\# & Frequency & Amplitude & Phase & \\
\hline$\underline{V} \mathrm{~F} 1$ & 13.2296329 & 6.66752952 & 0.60585 & \\
\hline$\sqrt{V} \mathrm{FZ}$ & 13.6935762 & 4.42120625 & 0.80427 & \\
\hline$\underline{F} \mathrm{~F} 3$ & 13.4807363 & 2.69991191 & 0.0444627 & \\
\hline V. F4 & 12.3970849 & 0.646561986 & 0.225613 & \\
\hline$\underline{V} \mathrm{~F} 5$ & 14.3167935 & 2.20250139 & 0.503499 & \\
\hline$\sqrt{V} \mathrm{~F} 6$ & 10.8652946 & 0.18698176 & 0.21971 & \\
\hline V F7 & 12.8327971 & 0.763452543 & 0.253063 & \\
\hline V] F8 & 14.6145332 & 1.36159004 & 0.745201 & \\
\hline V F9. & 12.1774095 & 0.595269379 & 0.436497 & \\
\hline V] F10 & 13.8076095 & 0.266180846 & 0.641807 & \\
\hline V F11 & 11.7502769 & 0.666158409 & 0.871134 & \\
\hline [V] $\mathrm{F} 12$ & 13.3488445 & 0.918091208 & 0.952598 & $=$ \\
\hline \multicolumn{2}{|c|}{ Calculate } & Improve all & Improve special & \\
\hline
\end{tabular}

Figure 4. The periodic time schift calculation mode.

will extend the program to handle time series of spectra. Those extensions will be part of a second release later this year.

The program is currently being tested and will be available for download soon. For more information on the current status of the program and the expected release date please visit the Period04 website: http://www.astro.univie.ac.at/ dsn/dsn/Period04 Comments and suggestions may be sent via email to: lenz@astro.univie.ac.at 


\section{Acknowledgements}

We would like to thank M. Sperl for valuable comments. This work is supported by the Austrian Fonds zur Frderung der wissenschaftlichen Forschung (Project P14546-PHY).

\section{References}

Bevington, P.R. 1969, Data reduction and error analysis for the physical sciences, McGraw-Hill (New York)

Breger, M. 1990, Comm. Asteroseismology 20,1

Sperl, M. 1998, Comm. Asteroseismology 111,1 